\title{
Numerical Simulation of an Unsteady Axisymmetric Flow in a Poppet Valve Using a Vortex Method
}

\author{
Tetsuhiro Tsukiji and Yoshikazu Suzuki \\ Department of Mechanical Engineering \\ Ashikaga Institute of Technology \\ 268-1 Oomae-cho, Ashikaga-city, Tochigi-prefecture, Japan
}

\begin{abstract}
In the present study, numerical simulations of the flow in a three-dimensional axisymmetric poppet valve are conducted using a vortex method. The fluids are assumed inviscid and incompressible. The vibration of the poppet, supported by a spring and a dash pot, occurs in the axial direction in some range of parameters. The numerical simulations are carried out by varying systematically the parameters of the calculation, namely the supply pressure, the spring constant, the mass of the poppet and the conical angle of the poppet. The process of jet flow development, the fluctuations of the valve lift and the velocity distributions in the valve are investigated for the poppet vibrating.
\end{abstract}

\section{Introduction}

At the present time, poppet valves are universally used as a control valve for hydraulic and pneumatic power applications. Because of the interest in the flow inside the poppet valve, there have been several studies of the flow in the valve by numerical simulations (Itoh et al. [6], Tsukiji [9], Vaughan et al. [12], Ueno et al. [11]). In those investigations, the valve lift was constant or there was no vibration of the poppet. Therefore, there has been only a small amount of information about the unsteady flow in presence of the vibration. The actual poppet valves sometimes vibrate in unstable regions of the operation. In the present numerical simulation the poppet can move in the axial direction because it is supported by a dash pot and a spring. Therefore the vibration of the poppet occurs in some range of parameters. The authors have calculated the axisymmetric unsteady flow in the poppet valve using a vortex method [10]. In the present study the same flow field is simulated using the axisymmetric vortex method to investigate the influence of the parameters including the conical angle of the poppet on the fluctuations of the valve lift and the flow in the valve.

The modelling of two-dimensional shear layers by discrete vortices has been studied by many researchers. In application to axisymmetric flows, internal and external flows, including jet flows, have been investigated using vortex methods (Acton [1], Edwards and Morfey [4], Chung and Troutt [3], Bernardinis et al. [2], Giovannini and Gagnon [5], Koso [7], Shimizu [8]). The results presented in this paper are obtained using a similar method.

In the present simulation, the source in the inlet plane is represented by a series of concentric rings of appropriate strength. The inlet boundary condition is satisfied by using image vortices

together with a source disk of uniform strength per unit area. The boundary walls such as the 
poppet and the valve body are approximated by a large number of vortex ring elements. The separated shear layer from the edge of the orifice is represented by an array of discrete elemental ring vortices. Each vortex element moves under the influence of all the others, including the set of image vortices and a source disk.

The process of jet flow development, the fluctuations of the valve lift and the velocity distributions in the valve were investigated, by means of a vortex method, for the unsteady flow when the poppet moves or vibrates in the axial direction.

\section{Method of simulation}

The axisymmetric poppet valve is shown in Fig. 1. The valve is a conical poppet with a sharp-edged seat. In the present simulation, flow passes through the valves in an outward direction and the conical angles $2 \alpha$ are $60^{\circ}$ and $120^{\circ}$.

A schematic of the flow simulation geometry of the part abcd in Fig. 1 is shown in Fig. 2. The inlet diameter is $d$, the radius of the valve chamber is $r_{\text {wall }}$, the valve lift is $h$ and the conical angle is $2 \alpha$. The line IJK shows the poppet surface and the lines ABCD and EFGH show the wall of the valve body. The annular jet is formed from the edges $\mathrm{C}, \mathrm{F}$ into a still environment. The numerical simulation starts with the moment that the flow first leaves the edges and follows the development of the ensuing jet. The continuous vortex sheet is represented by an array of discrete elemental ring vortices. As each vortex element leaves the edges it is replaced at the edges by a new element. The poppet surface IJK and the wall of the valve chamber ABCD and EFGH are represented by the number of vortex rings located with equal spacing. The total number of ring elements is kept constant $(M)$ during the simulation. The source in the inlet plane DE is represented by a series $(L)$ of concentric source rings of appropriate strength. Image vortices and a source disk of uniform strength are used to satisfy the imposition of the normal velocity and the inlet boundary.

As the axisymmetric jet is assumed, the flow field ABCDOJI is used to explain the numerical method.

The induced axial and radial velocities for the $k$-th vortex are given by

$$
\begin{gathered}
u_{k}=\sum_{\substack{i=1 \\
i \neq k}}^{N} \gamma_{i} u_{v i}-\sum_{i=1}^{N} \gamma_{i} u_{v i}^{*}+\sum_{i=1}^{M} \gamma_{w i}\left(u_{v i}-u_{v i}^{*}\right)+\sum_{i=1}^{L} q u_{s i}+u_{s e l f, k} \\
v_{k}=\sum_{\substack{i=1 \\
i \neq k}}^{N} \gamma_{i} v_{v i}-\sum_{i=1}^{N} \gamma_{i} v_{v i}^{*}+\sum_{i=1}^{M} \gamma_{w i}\left(v_{v i}-v_{v i}^{*}\right)+\sum_{i=1}^{L} q v_{s i}
\end{gathered}
$$

where $u_{v i}$ and $v_{v i}$ are the axial and radial velocities induced by the vortices located on the surface of the valve chamber and poppet [8]. $u_{s i}$ and $v_{s i}$ are the axial and radial velocities induced by the sources located on the inlet plane. The superscript ${ }^{*}$ shows the velocity induced by the image vortices. $\gamma_{i}$ and $\gamma_{w i}$ show the strength of the vortices representing the shear layer, and the walls of the poppet and the valve chamber, respectively. $q$ is the strength of the concentric source ring on the inlet plane.

The self-induced velocity of $k$-th vortex is given by

$$
u_{s e l f, k}=\frac{\gamma_{k}}{4 \pi R_{i}}\left(\ln \frac{8 R_{i}^{3 / 2}}{B}-.25\right)
$$


where $\gamma_{k}$ is the vortex strength, $R_{i}$ is the radius of the vortex ring and the constant $B$ is chosen 0.001 which was used by Acton [1].

The flow rate $Q$ is determined by the strength of all concentric source rings.

Associated with a unit strength of each elemental vortex ring there is a velocity field, whose axial and radial components at a point $(x, y)$ are given by the following equations (Shimizu [8], Edwards [4]):

$$
\begin{aligned}
& u_{v}=R_{i}\left(R_{i} J-y G\right) \\
& v_{v}=\left(x-X_{i}\right) R_{i} G
\end{aligned}
$$

The axial and radial velocity components induced by a unit strength of concentric source ring at a point $(x, y)$ are respectively

$$
\begin{aligned}
& u_{s}=\left(x-X_{i}\right) J \\
& v_{s}=\left(y J-R_{i} G\right)
\end{aligned}
$$

where $X_{i}$ is the axial coordinate of the vortex or source ring, $R_{i}$ is the radius of the ring,

$$
\begin{gathered}
r^{2}=\left(x-X_{i}\right)^{2}+\left(y+R_{i}\right)^{2} \\
k=\frac{4 y R_{i}}{r^{2}} \\
J=\frac{1}{\pi r^{3}} \times \frac{E(k)}{1-k^{2}} \\
G=-\frac{1}{\pi r^{3}} \times \frac{1}{k}\left[2 K(k)-\frac{2-k^{2}}{1-k^{2}} E(k)\right]
\end{gathered}
$$

and $K(k), E(k)$ are complete elliptic integrals of the first and second kind respectively. The values $K(k), E(k)$ were calculated using the method presented by Koso [7] to reduce a computational time.

The boundary conditions on the walls are given by

$$
\begin{aligned}
u & =0 \quad(\mathrm{BC}) \\
v & =0 \quad(\mathrm{AB}, \mathrm{CD}) \\
u_{p} \tan \alpha-v_{p} & =U \tan \alpha \quad(\mathrm{IJ})
\end{aligned}
$$

where $U$ is the velocity of the poppet and $u_{p}, v_{p}$ are the velocity components in the $x, y$ directions on the boundary IJ.

The strength of the vortex ring $\gamma_{w i}$ can be calculated by solving the simple simultaneous equations obtained from Eqs. 1, 2, 12, 13 and 14. The vortex element at the edge $\mathrm{C}$ leaves for a flow field with an axial velocity component calculated by Eq. 1 and the vortex ring $\gamma_{i}$ representing the separated shear layer is introduced. The new axial and radial positions of each vortex ring can be obtained to first-order approximation. Any of the vortices that approached closer than a value $\delta$ to the wall and flowed through the boundary AI are removed from the calculation. The removing of vortices near the wall is due to the viscosity of the boundary layer near the wall. It does not 
happen frequently. The calculation is performed until the total number of vortex elements inside the computational domain remains approximately constant.

The pressure distributions on the poppet were calculated using the unsteady Bernoulli's equation

$$
\frac{\partial \phi}{\partial t}+\frac{V^{2}}{2}+\frac{p}{\rho}=f(t)
$$

where $\phi$ is the velocity potential, $V$ is the magnitude of the velocity, $p$ is the pressure, $t$ is the time and $\rho$ is the density. The pressure can be calculated using the equation obtained by differentiating Eq. 15 with respect to the distance. The upstream pressure is assumed to be constant.

The equation of motion for the poppet is given by

$$
F=m \frac{\partial^{2} h}{\partial t^{2}}+c_{f} \frac{\partial h}{\partial t}+k h
$$

where $F$ is the force acting on the poppet, $h$ is the valve lift, $m$ is the mass of the poppet, $c_{f}$ is the coefficient of viscosity and $k$ is the spring constant.

The numerical implementation proceeds for each time step as follows.

1. Determine the strength of the vortex rings on the walls of the poppet and the valve chamber.

2. Calculate the pressure distribution using Eq. 15.

3. Calculate the force acting on the poppet by integrating the pressure distribution.

4. Move the poppet by calculating the valve lift at $t+\Delta t$ using Eq. 16 .

5. Introduce the vortex ring $\gamma_{i}$ and advance the vortices in time.

6. Delete any vortices that approach closer than $\delta$ to a wall or pass through the outlet boundary.

7. Return to step 1.

\section{Results and discussion}

In the present calculation, $L=5, M=53, \delta=0.1 \mathrm{~mm}, t=0.02 \mathrm{msec}$ and the spacing of the vortex elements on the wall is $d / 6$. The values of $d, r_{\text {wall }}, \alpha$ and $\overline{\mathrm{AB}}$ were selected based on the interests of practical applications in order to compare with experimental results. The following parameters were used: $d=10 \mathrm{~mm}, r_{\text {wall }}=20 \mathrm{~mm}, \alpha=30^{\circ}$ and $\overline{\mathrm{AB}}=20 \mathrm{~mm}$.

The values of the supply pressure $p_{u p}$, the spring constant $k$ and the mass of the poppet $m$ in the present study are shown is Table I.

The viscosity coefficient $c_{f}$ was evaluated from the clearance and the contact area between the poppet and the valve body. As a result, $c_{f}=2.258 \mathrm{~Pa}$ was chosen.

In the present calculation, it is assumed that the supply pressure $p_{u p}$ is constant. Therefore, after the steady flow rate at a valve lift was given by experimental results, the flow rate (the strength of the concentric source ring $q$ ) at each calculation step was given in proportion to the valve lift.

The valve lift of the poppet in cases A-D of Table I are shown in Fig. 3 for $2 \alpha=60^{\circ}$. The poppet in the cases $\mathrm{A}$ and $\mathrm{B}$ vibrate near the valve lift $h=6 \mathrm{~mm}$ for $t \geq 100 \mathrm{msec}$. In case A, a transient response with overshoot can be seen. However almost no overshoot occurs in case B because the mass of the poppet in case B is smaller than that in case A. Furthermore, the amplitude of vibration in case $\mathrm{B}$ is larger than that in case $\mathrm{A}$ for $t \geq 150 \mathrm{msec}$ because of the same reason. 
Table I: Calculation parameters $\left(2 \alpha=60^{\circ}, 120^{\circ}\right)$

\begin{tabular}{|c|c|c|c|}
\hline Case & Supply pressure $p_{u p}[\mathrm{MPa}]$ & Spring constant $k[\mathrm{~N} / \mathrm{cm}]$ & Valve mass $m[\mathrm{~kg}]$ \\
\hline A & 0.14 & 325.9 & 0.407 \\
\hline B & 0.14 & 325.9 & 0.051 \\
\hline C & 0.14 & 1303 & 0.407 \\
\hline D & 0.05 & 325.9 & 0.407 \\
\hline
\end{tabular}

The poppet in the cases $\mathrm{C}$ and $\mathrm{D}$ vibrate near $h=1-2 \mathrm{~mm}$ because the force acting on the poppet in the direction to close the valve is large as compared with the cases $\mathrm{A}$ and $\mathrm{B}$. The frequencies of vibration of the poppet are $100 \mathrm{~Hz}$ and $55 \mathrm{~Hz}$ for the cases $\mathrm{C}$ and $\mathrm{D}$, respectively, and the natural frequencies of the poppet for these cases are $90 \mathrm{~Hz}$ and $45 \mathrm{~Hz}$, respectively. Therefore, it is found that the poppet in the cases $\mathrm{C}$ and $\mathrm{D}$ vibrate near their natural frequencies.

The trajectories of the vortex rings and the velocity vectors for case A are displayed in Fig. 4 between $t=2 \mathrm{msec}$ and $t=8 \mathrm{msec}$. The development of the starting free jet and the vortex clusters formed by many vortex ring elements into a still environment can be seen. A large vortex is also found in the flow at $t=8$ msec.

The valve lifts in the cases $\mathrm{A}$ and $\mathrm{C}$ at dimensional times from $t=180 \mathrm{msec}$ to $t=200 \mathrm{msec}$ are shown in Figs. 5 and 6 . The circles $\mathrm{O}$ show the time when a vortex cluster is generated near the valve seat and squares $\square$ show the time when a cluster reaches the outlet AI. In Figs. 5 and 6 , the number of circlea is almost the same, however the time interval for the generation of a cluster is not constant. The number of squares is smaller than that of circles in Fig. 6, because of the coalescence of the cluster. The process of coalescence of clusters is shown in Fig. 7.

A magnified figure of the valve lift in case A for $150 \mathrm{msec}<t<200 \mathrm{msec}$ is shown in Fig. 8 while the experimental results for the same case is shown in Fig. 9. The profiles of the time variation of the valve lifts are similar. However the amplitude of the variation of the experimental results is smaller than the calculated ones because the viscous resistance in the actual poppet valve is large as compared with the expected value in the present calculation.

The valve lifts of the poppet in cases A-D shown in Table I are shown in Fig. 10 for $2 \alpha=120^{\circ}$. It is found that the amplitude of the vibration for $2 \alpha=120^{\circ}$ is larger than that for $2 \alpha=60^{\circ}$ from Figs. 3 and 10.

The trajectories of the vortex rings and the velocity vectors in case A are displayed in Fig. 11 for $2 \alpha=120^{\circ}$ between $t=2 \mathrm{msec}$ and $t=8 \mathrm{msec}$.

\section{Conclusion}

In the present study, a numerical algorithm using a vortex method has been applied to the simulation of the flow in a three-dimensional axisymmetric poppet valve supported by a dash pot and a spring. The process of jet flow development, the fluctuations of the valve lift and the velocity distributions in the valve were shown with the vibration of the poppet. The numerical simulation were carried out by varying systematically the parameters of the calculation, namely the supply pressure, the spring constant, the mass of the poppet and the conical angle of the poppet. The effect of those parameters on the time variation of the valve lift and the formation of the vortex cluster was investigated. It is found that the poppet vibrates near its natural frequency. Comparison of the time variations of the valve lift in case A has shown the difference between the numerical and experimental results. This is probably related to the difference between the viscous resistance 
of the actual valve and the expected resistance in the calculation. Furthermore the poppet whose conical angle is large vibrates with large amplitude.

\section{Acknowledgments}

We would like to thank Prof. S. Shimizu of the Hiroshima Institute of Technology for many helpful discussions and comments. Thanks are also due to the Information and Science Center of the Ashikaga Institute of Technology for the present calculations.

\section{References}

[1] Acton, E., "A Modeling of Large Eddies in an Axisymmetric Jet," J. Fluid Mech., 98 (1), pp. $1-31,1980$.

[2] Bernardinis, B.D., Graham, J.M.R. and Parker, K.H., "Oscillatory Flow around Disks and through Orifices," J. Fluid Mech., 102, pp. 279-299, 1981.

[3] Chung, J.N. and Troutt, T.R., "Simulation of Particle Dispersion in an Axisymmetric Jet," J. Fluid Mech., 186, pp. 199-222, 1988.

[4] Edwards, A.V.J. and Morfey, C.L., "A Computer Simulation of Turbulent Jet Flow," Comp. and Fluids, 9, pp. 205-221, 1981.

[5] Giovannini, A. and Gagnon, Y., "Numerical Simulation of an Unsteady Axisymmetrical Orifice Plate Flow with a Perturbed Inlet velocity," Proc. FLUCOME'94, 1, pp. 407-412, 1994.

[6] Itoh, K., Takahashi, K. and Inoue, J., "Flow in a Poppet Valve (A Method for Computing Pressure Distribution using a Streamline Coordinate System)," Trans. Jpn. Soc. Mech. Eng., (in Japanese), 57 (539 B), pp. 2225-2232, 1991.

[7] Koso, T., "An Analysis of Round Jet using the Axisymmetric Discrete-Vortex-Method," Symp. Prepr. of Jpn. Soc. Mech. Eng., (in Japanese), 68 (890), pp. 37-40, 1992.

[8] Shimizu, S., "Discrete-Vortex Simulation of an Axisymmetric Flow in a Disk Valve," Prepr. of Jpn. Soc. Mech. Eng., (in Japanese), 68 (920), pp. 385-387, 1992.

[9] Tsukiji, T., "Discrete-Vortex Analysis for Tow-Dimensional Jet issuing from Poppet Valve Orifices," Research Rept. Ashikaga Inst. Tech., No. 17, pp. 7-14, 1991.

[10] Tsukiji, T. and Suzuki, Y., "A Computer Simulation of Flow in an Axisymmetric Poppet Valve Using the Vortex Method," Experimental and Numerical Flow Visualization, ASME, FED-218, pp. 103-108, 1995.

[11] Ueno, H., Okazima, A., Tanaka, H. and Hasegawa, T., "Noise Measurement and Numerical Simulation of Oil Flow in Pressure Control Valve," Trans. Jpn. Soc. Mech. Eng., (in Japanese), 58 (547 B), pp. 743-749, 1992.

[12] Vaughan, N.D., Johnston, D.N. and Edge, K.A., "Numerical Simulation of Fluid Flow in Poppet Valves," Proc. Instn. Mech. Engrs., 206, pp. 119-127, 1992. 


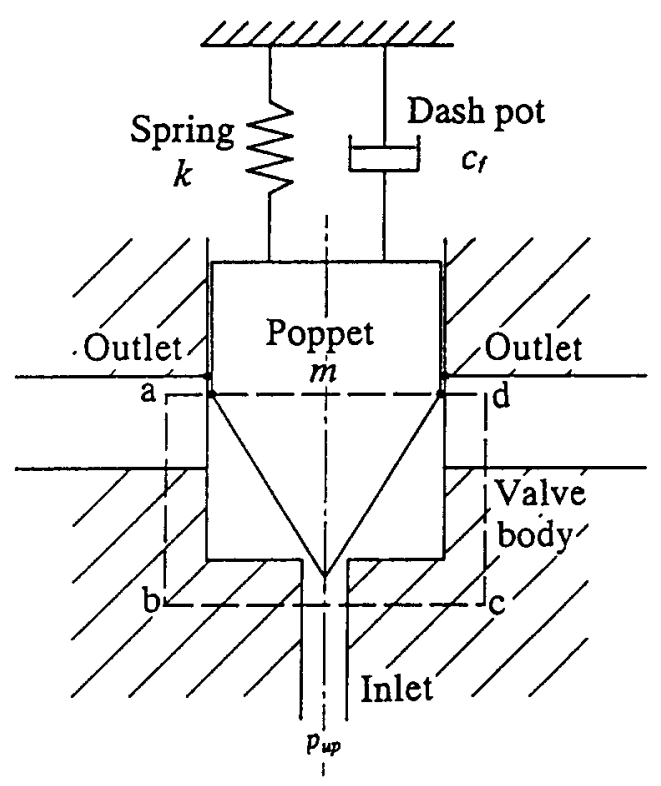

Figure 1: Poppet valve model.

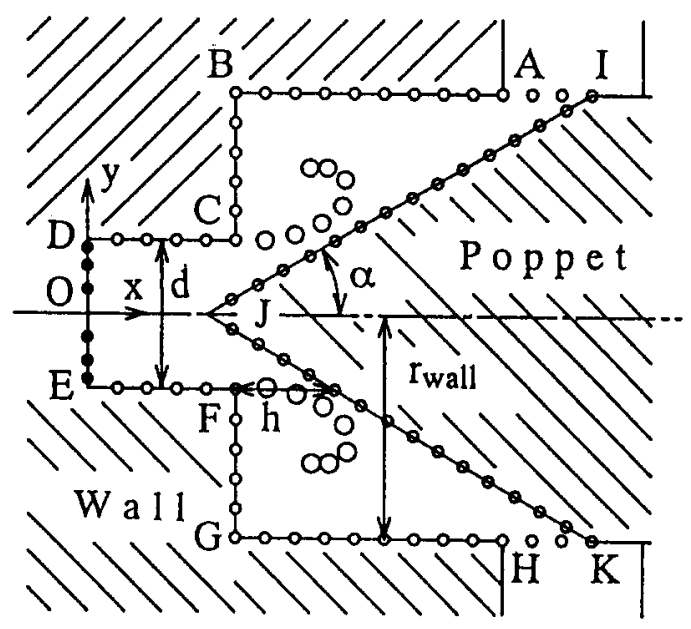

Figure 2: Flow simulation geometry. 


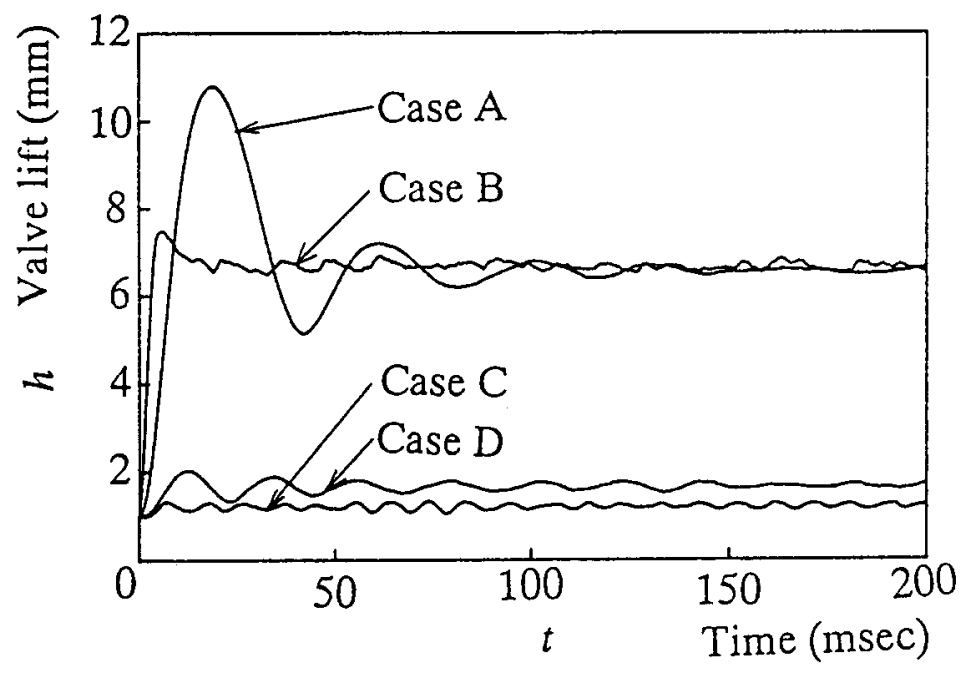

Figure 3: Valve lifts $\left(2 \alpha=60^{\circ}\right)$. 

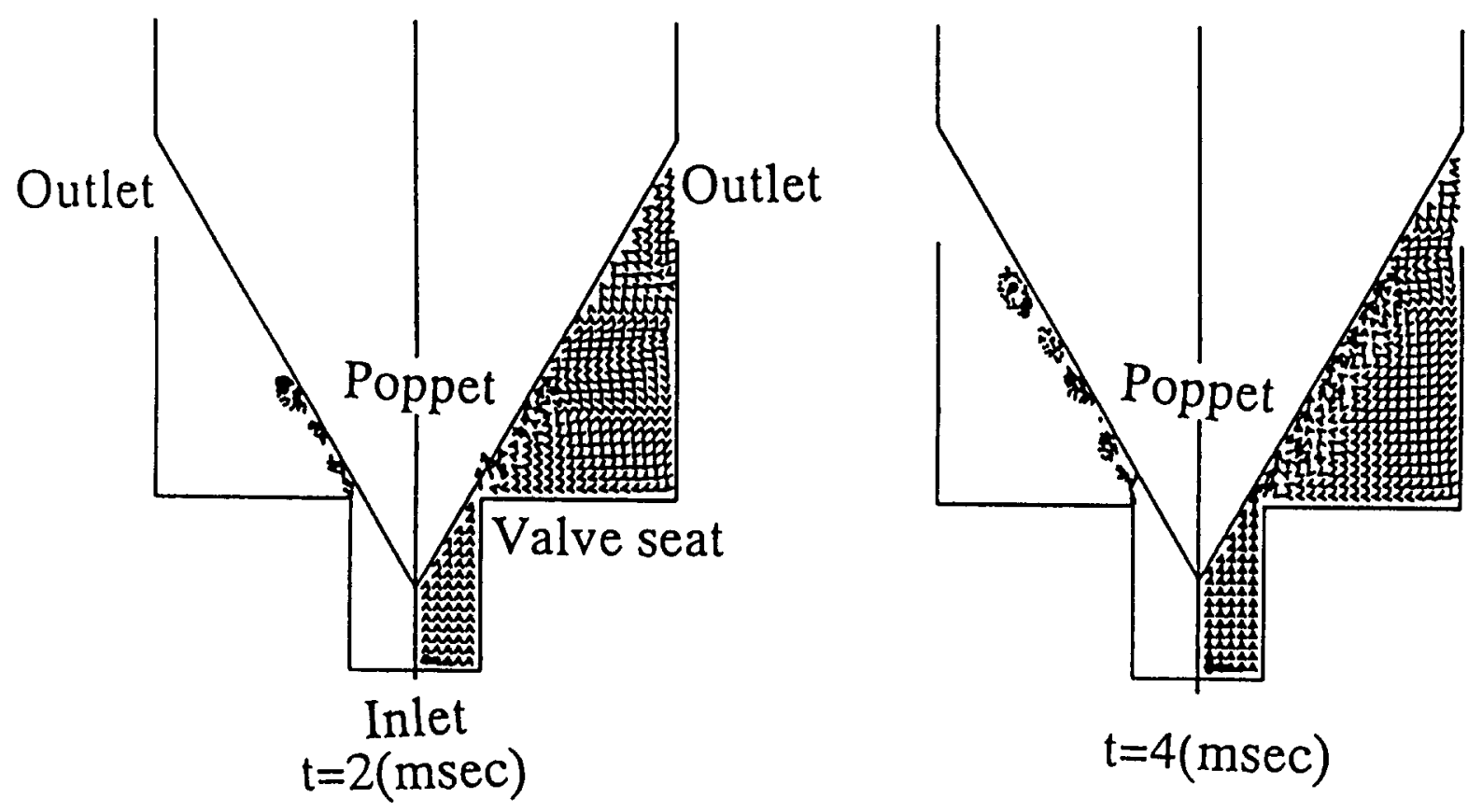

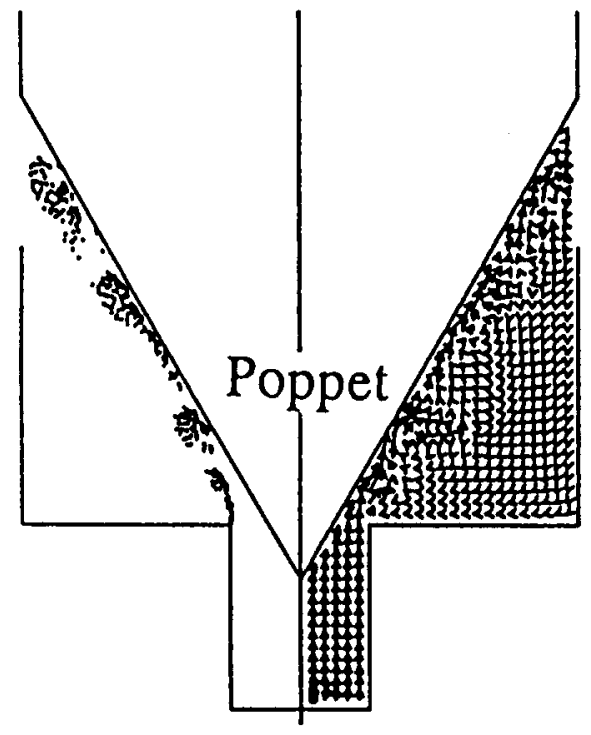

$\mathrm{t}=6(\mathrm{msec})$

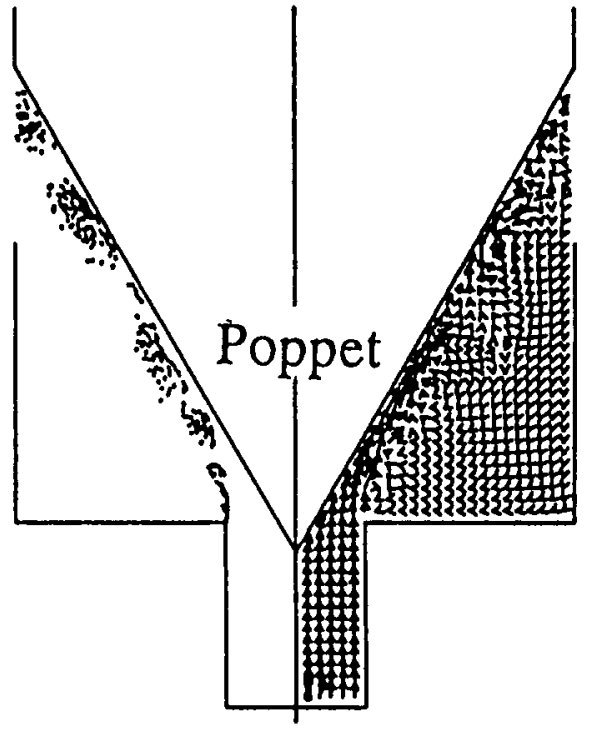

$\mathrm{t}=8(\mathrm{msec})$

Figure 4: Trajectories of vortex ring and velocity vectors (Case A, $2 \alpha=60^{\circ}$ ). 


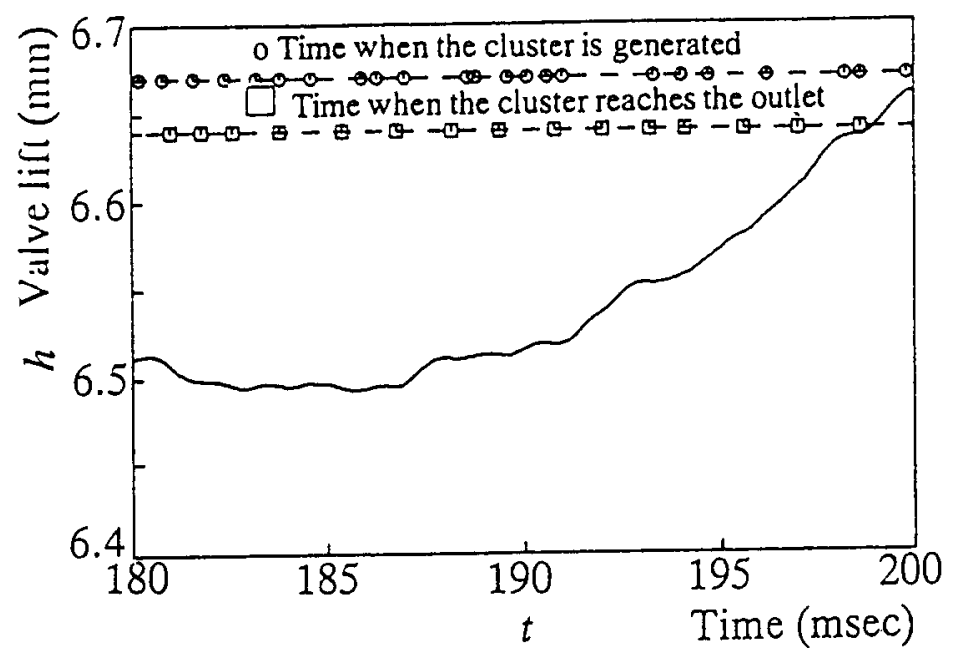

Figure 5: Valve lifts (case A, $2 \alpha=60^{\circ}$ ).

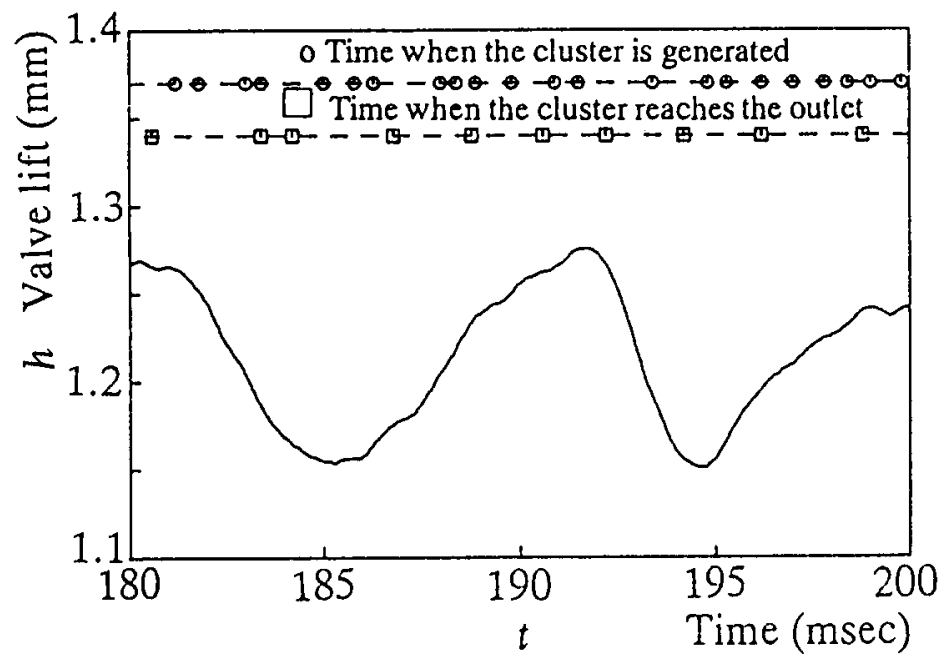

Figure 6: Valve lifts (case C, $2 \alpha=60^{\circ}$ ). 

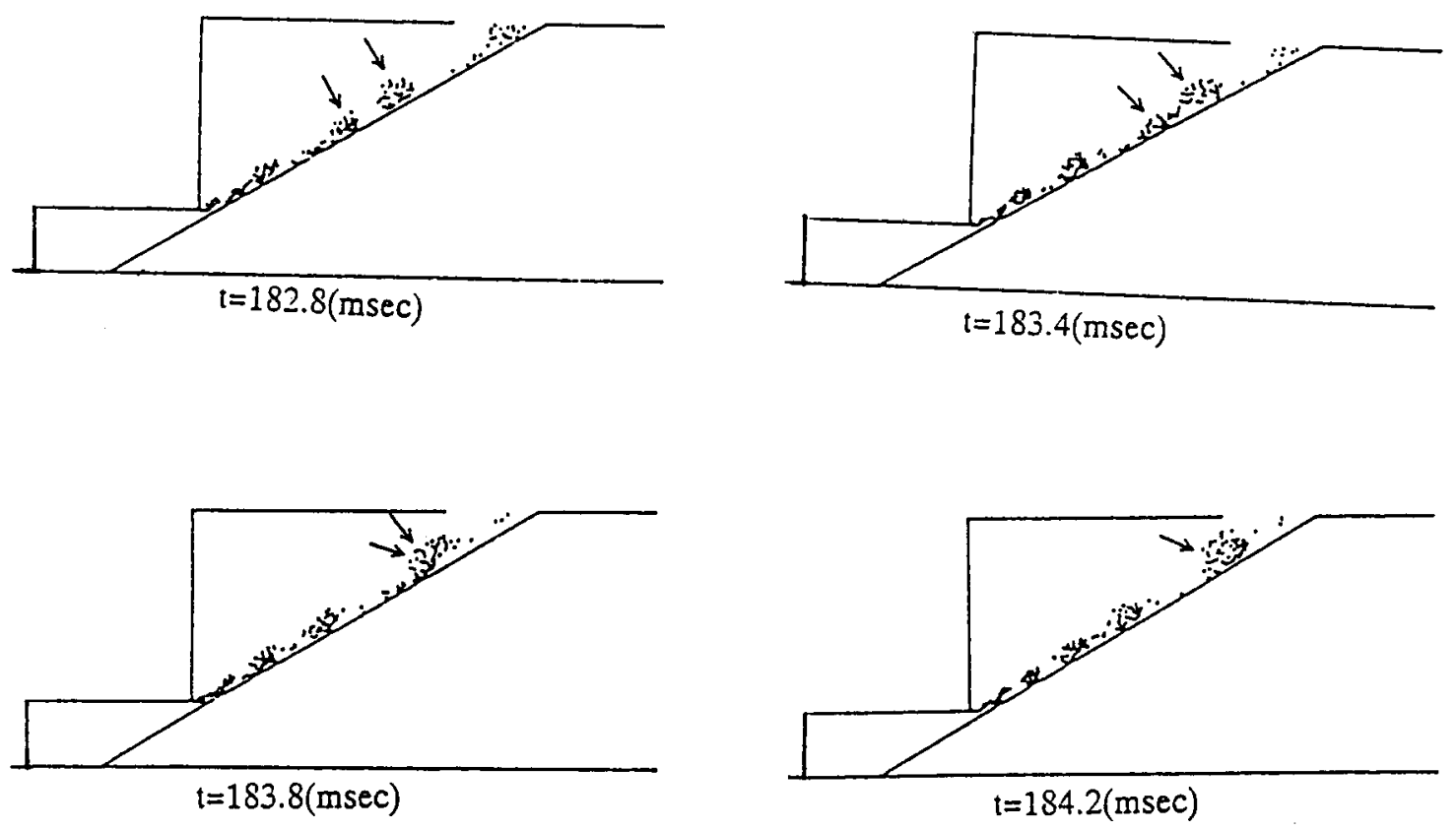

Figure 7: Trajectories of vortex rings (case $\left.\mathrm{C}, 2 \alpha=60^{\circ}\right)$.

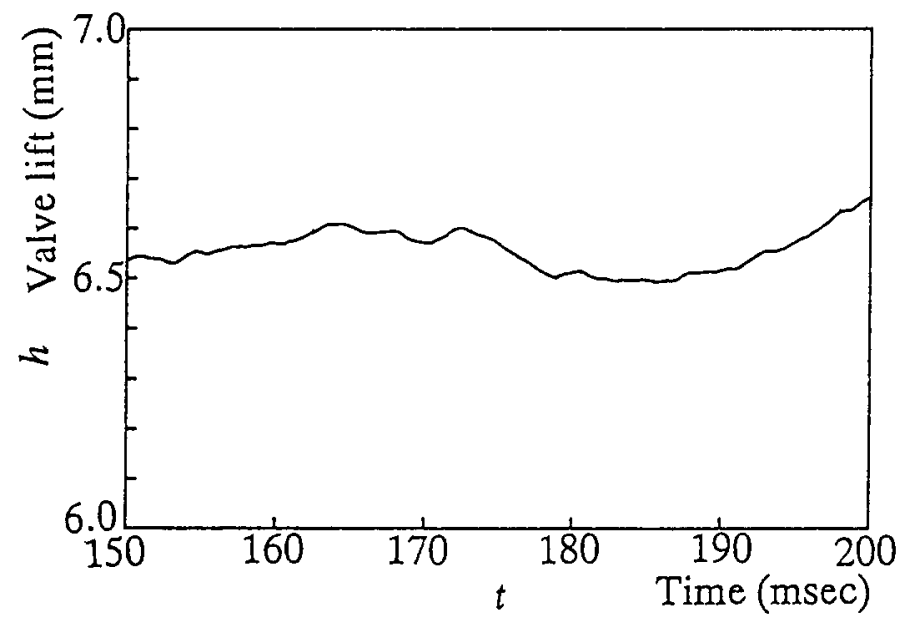

Figure 8: Valve lift (calculated result (Case A, $2 \alpha=60^{\circ}$ ). 


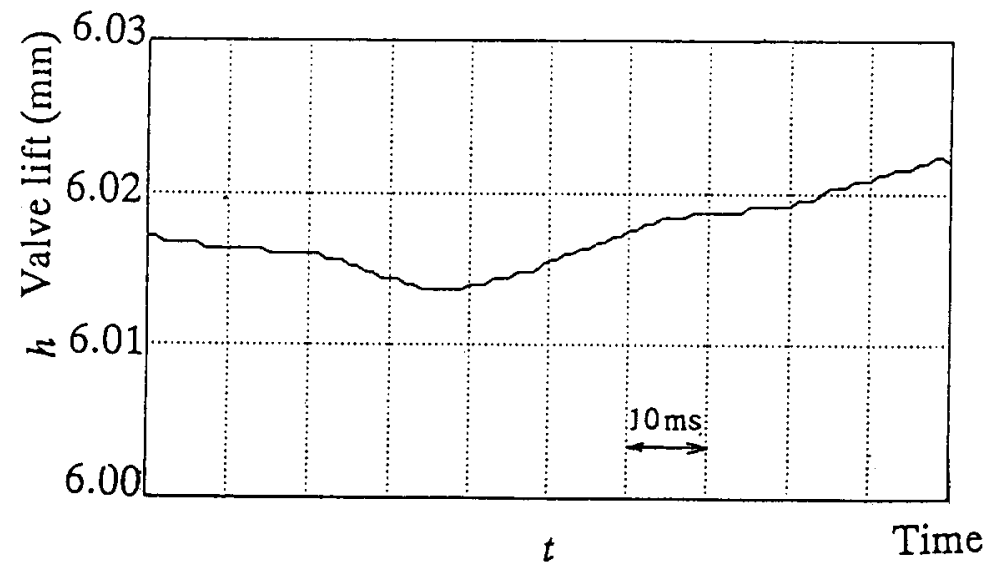

Figure 9: Valve lift (experimental result, $2 \alpha=60^{\circ}$ ).

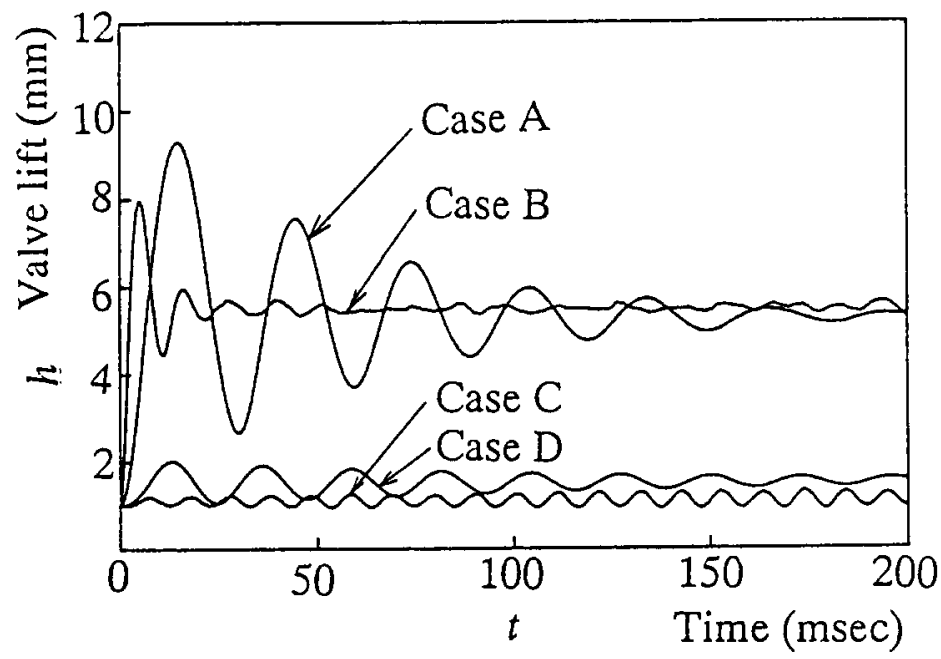

Figure 10: Valve lifts $\left(2 \alpha=120^{\circ}\right)$. 

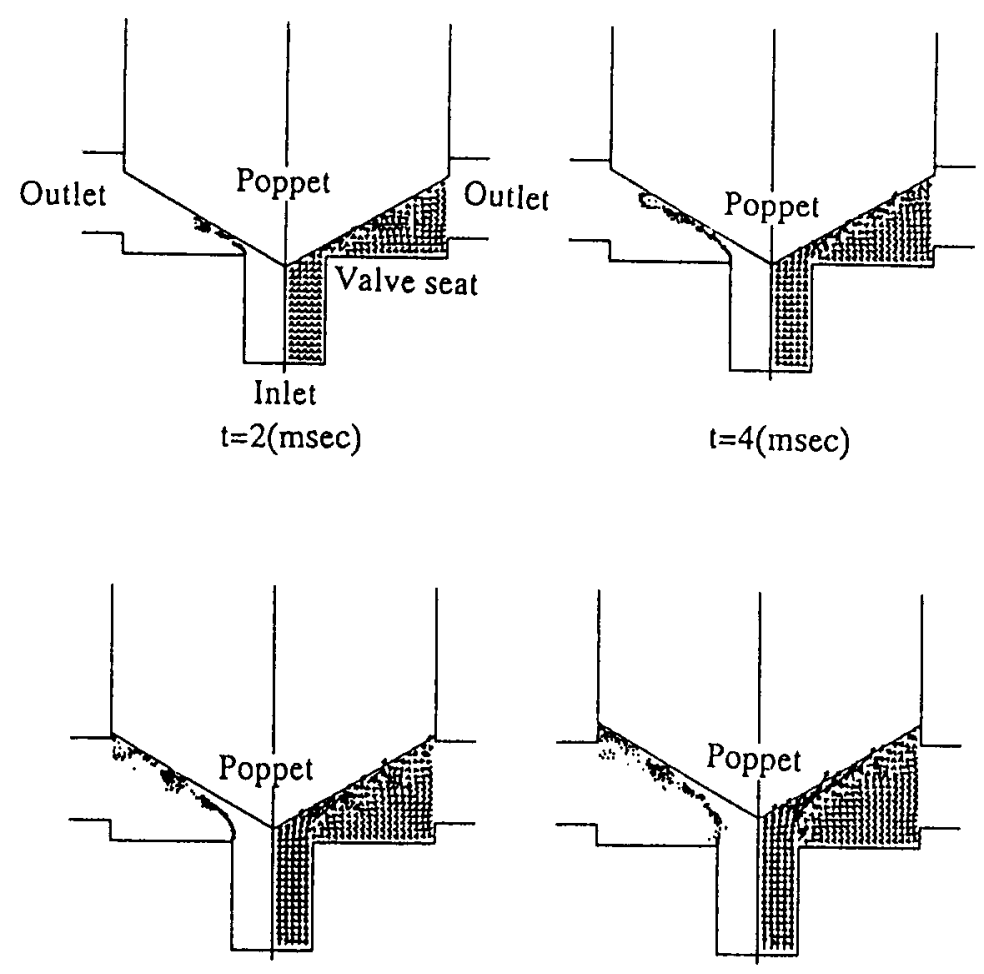

$\mathrm{t}=6(\mathrm{msec})$

$\mathrm{t}=8(\mathrm{msec})$

Figure 11: Trajectories of vortex ring and velocity vectors (Case A, $2 \alpha=120^{\circ}$ ). 